\title{
A lentinan-loaded hydrogel with core-shell structure induces broad-spectrum resistance against plant virus by activating the expression of CML30
}

\author{
Shunyu Xiang ${ }^{1}$, Xue Chen ${ }^{1}$, Jing Wang $^{1}$, Haoran Peng ${ }^{1}$, Xing Lv ${ }^{1}$, Long Jiang ${ }^{1}$, Zhe Cao ${ }^{1}$, \\ Jin Huang ${ }^{1}$, Daibin Wang ${ }^{2}$, Lisong $\mathrm{Ma}^{3}$, and xianchao sun ${ }^{1}$ \\ ${ }^{1}$ Southwest University \\ ${ }^{2}$ Chongqing Tobacco Science Research Institute \\ ${ }^{3}$ Agriculture and Agri-Food Canada
}

November 10, 2020

\begin{abstract}
Control of plant virus disease largely depends on the induced plant defense achieved by the external application of synthetic chemical inducers with the ability to modify defense-signaling pathways. However, most of the molecular mechanisms underlying these inducers remain unknown. Here, we developed a lentinan-loaded hydrogel with the core-shell structure and discovered how it protects plant from different virus infections. The hydrogel was synthesized by adding a chitosan shell on the surface of the sodium alginate-calcium ion-lentinan (LNT) hydrogel (SL-gel) to form CSL-gel. CSL-gel exhibits the capacity of prolonging the stable-release of lentinan and promoting calcium ions release. Application of CSL-gel on the root of plants significantly promotes plant growth and development and induces broad-spectrum resistance against TMV, TuMV, PVX and TRV. Furthermore, we found that the sustained release of calcium ions from the CSL-gel triggers the high expression of cal-modulin-like protein 30 (CML30), and silencing of CML30 enhances the susceptibility of tobacco to TMV. Our findings provide evidence that the novel and synthetic CSL-gel with the sustainable release of LNT and calcium ion strongly inhibits the plant virus infection. This study uncovers a novel mode of action by which CSL-gel with the stable release of calcium ion triggers CML30 expression.
\end{abstract}

\section{Introduction}

Inducing and activating plant immune response is one of the most effective ways to combat plant virus diseases ${ }^{[1]}$. Plant virus that has a variety of infection modes, a wide range of host and a long infection cycle causes a common systemic infection ${ }^{[2]}$. Control of plant virus disease largely depends on the application of the virus passivation agents. However, these agents are not able to provide complete control of the plant viral diseases owing to their ability to partially reduce the virus infection and inhibiting the replication and transfer of the virus in the plant ${ }^{[3]}$. Inducers with the ability to activate plant resistance are becoming an increasingly important way to control plant virus disease. Previous studies showed that they can significantly induce the expression of resistance-related genes and enhance the immune response of plants to biotic stresses $^{[4]}$. However, the application of the existing inducers in the field is limited because their lasting time is shorter than the existing period of virus infection. In addition, most of the inducers consist of biological polysaccharides with a structure that is easily degraded at the complicated environment in the field, rustling in the reduced efficacy on the control of virus disease ${ }^{[5-6]}$. Therefore, there is an urgent need to develop a novel inducer agent with the ability to last the release time of loaded drugs for the field application.

Calcium ions $\left(\mathrm{Ca}^{2+}\right)$ as an essential nutrient element, can promote plant growth, improve photosynthesis, and increase the synthesis and accumulation of organic matter ${ }^{[7]} \cdot \mathrm{Ca}^{2+}$ as a second messenger is also involved 
in the signal transduction of plant to stress responses ${ }^{[8-9]}$. Previous studies showed that $\mathrm{Ca}^{2+}$ signals are perceived by four types of calcium signaling sensor proteins, including calmodulin ( $C a M$ ), calmodulin-like protein $(C M L)$, calcium-dependent protein kinase (CDPK), and calcium-like protein Calcineurin B-like $(\mathrm{CBL})^{[10-11]}$. However, $C M L, \mathrm{CDPK}, \mathrm{CBL}$ are found only in plants and some protists. $C M L s$, as one of the plant-specific $\mathrm{Ca}^{2+}$ receptors, participates in plant biological stress, abiotic stress as well as many developmental processes ${ }^{[12-13]}$. It has been documented that plant defense response, plant growth and development as well as cytokine regulation, induce changes in intracellular calcium ion concentration, which results in the induced expression of $C M L s$ gene ${ }^{[14-15]}$. Accumulated evidence showed that the up-regulation of $C M L$ gene expression is able to significantly improve the plant resistance to biotic stress ${ }^{[16-17]}$. For example, $C M L 41$ was found to reduce $P$. syringae infection by regulating plasmodesmal closure through mediating a $\mathrm{Ca}^{2+}$ signaling in response to bacterial pathogens ${ }^{[17]}$. Leba et al. showed that $C M L 9$ is involved in plant resistance to pathogenic bacteria through a flagellin-dependent pathway in plants ${ }^{[18]}$. Tobacco calmodulinlike protein, rgs-CaM, binds to the RNA silencing (RNAi) suppressor of the tobacco etch virus to inhibit RNAi and increase tobacco resistance to the virus ${ }^{[19]}$. CML30 was found to be regulated by abiotic stress and play diverse roles in plant hormone synthesis and disease defense ${ }^{[20]}$. Therefore, $C M L$ can be regarded as an important target site to stimulate plant defense response.

Recent studies showed that sustained-release hydrogel as a drug carrier has attracted more interest in delivering therapeutic agents to control human diseases [21]. Advantages of hydrogel include a prolonged drug release and action time, increased stability of loaded drugs and enhanced control efficiency of disease ${ }^{[22-23]}$. In addition, many studies showed that sustained-release hydrogel exhibits the ability of carrying multiple drugs and providing various control of multiple diseases. For example, porphyrin photosensitizer sinoporphyrin sodium (DVDMS) and Polylactic acid-copolymerized glycolic acid (PLGA) are loaded into the sodium alginate-chitosan hydrogel that provides the dual action of antibacterial and skin regeneration ${ }^{[24]}$. Therefore, the development of dual functions of hydrogel that could deliver a range of drugs and provide the broad-spectrum resistance to multiple diseases is required.

Our previous studies found that coating the surface of hydrogel with amino oligosaccharides can prolong the release time of drugs loaded in the hydrogel (ALA-gel) ${ }^{[25]}$. However, amino oligosaccharides are highly soluble in water, resulting in the slow dissolve of the ALA-gel surface and the reduced drug release time from the ALA-gel. To overcome the drawbacks of ALA-gel and improve the sustained release of drugs loaded in the hydrogel, a multiple-functional sodium alginate-lentinan hydrogel (SL-gel) with a dense chitosan shell (CSL-gel) was developed aiming at improving the control efficacy against plant virus diseases. As expected, CSL-gel exhibited stable and sustained LNT release and broad-spectrum anti-virus activities. The sustainable and controlled release of calcium ions activated the expression of CML30 that enhances the resistance of tobacco against virus with a lasting time up to 30 days after CSL-gel treatment.

\section{Materials and Methods}

\section{Chemical reagents}

The following chemical materials and reagents were used and purchased from commercial suppliers without further purification: SA and chitosan (viscosity of $200 \pm 20 \mathrm{mPas}$, Shanghai Aladdin Biochemical Technology Co., Ltd., Shanghai, China); $\mathrm{CaCl}_{2}$ (Kaixiang fine chemical co. LTD, Shanghai, China); LNT (purity:98\%, Watson International Ltd).

\section{Synthesis of CSL-gel}

The SA, $\mathrm{CaCl}_{2}$, lentinan (LNT) and chitosan were used to synthesis the CSL-ge following the method as described previously ${ }^{[25-26]}: 1 \mathrm{~g} \mathrm{SA}$ and $1 \mathrm{~g}$ LNT were dissolved in $125 \mathrm{~mL}$ of distilled water and stirred until completely dissolved. Subsequently, the SA-LNT mixed solution was slowly dropped into $1000 \mathrm{~mL}$ of aqueous $\mathrm{CaCl}_{2}(30 \mathrm{~g} / \mathrm{L})$ solution (stirred at 400 revolutions/min for $30 \mathrm{~min}$ ) to form a hydrogel precursor (SL-ge) immediately (loading efficiency ${ }^{[27]}$ of $15.2 \%$ and encapsulation percentage ${ }^{[27]}$ of $89.4 \%$ ). Next, $10 \mathrm{~g} / \mathrm{L}$ aqueous chitosan solution was added to the surface of the SL-gel and stirred for $2 \mathrm{~h}$ (400 revolutions/min) to form the CSL-gel. The CSL-gel was washed three times with deionized water. 


\section{Characterization of the chitosan core-shell of CSL-gel}

Scanning Electron Microscopy (SEM), Zeta potential analysis, Fourier Transform infrared spectrometry (FTIR) and elemental analysis were performed by Beijing Zhongke Baitei Technical Service Co., LTD. Briefly, SEM (SU-8020, Hitachi, Japan) was used for the observation of the surface composition of the hydrogel according to manufactories instruction. Zetasizer Nano ZS90 (Malvern Panalytical, Almelo, Netherlands) was used to measure the surface charge of the hydrogel. FTIR spectrometer Nicolet 6700 (Thermo Scientific, USA) was used to collect infrared spectra in the range $4000-400 \mathrm{~cm}-1$ by 32 scans following the manufacturer's instructions. Elemental analyzer (Vario EL cube, Elementar Analysensysteme Madison, WI, U.S.A, Frankfurt, Germany) were used to analyze the elemental composition on the formation of the chitosan shell in the surface of the hydrogel.

\section{Measurement of LNT and calcium ion release from CSL-gel}

The standard curve of LNT was determined by UV-vis spectrophotometry (UV-vis, PGEN-ERAL) according to the method as described previously ${ }^{[25]}$. The sustained-release ability and rate of CSL-gel and SL-gel were tested according to the previous methods ${ }^{[25]}$ : Briefly, 50 pieces of CSL-gel or SL-gel were added into $10 \mathrm{~mL}$ of deionized water ( $\mathrm{pH} 5$ ) at $20 \mathrm{degC}$ and an UV-vis spectrophotometer was used to measure the absorbance of LNT in solution at 24-336 h, respectively. The same method was used to measure the cumulative release of calcium ions and investigate the effects of different temperatures (Temperature: 10, 20, 30, 40, 50, and 60 $\operatorname{deg} \mathrm{C}, \mathrm{pH}=5)$, different $\mathrm{pH}$ values ( $\mathrm{pH}: 3.0,5.0,7.0$, and 9.0, adjusted by Tris- $\mathrm{HCl}$, at $20 \operatorname{deg}$ ), and different concentrations of cations (The concentrations of $\mathrm{NaCl}$ solution were $0.1,0.2,0.4$, and $0.8 \mathrm{~mol} / \mathrm{L}$, respectively, $\mathrm{pH}=5$, at $20 \operatorname{deg} \mathrm{C}$ ) on the sustained-release ability and rate of CSL-gel as well. The cumulative release rate (CRR) of LNT was calculated according to formula 3. The release kinetics of LNT from CSL-hydrogel and SL-hydrogel were analyzed using Higuchi (Formula 4) and Korsmeyer-Peppas models (Formula 5) [28].

$\operatorname{CRR}(\%)=\frac{\sum_{0}^{t} C_{t} V_{\text {total }}}{Q_{1}} \times 100 \%(3)$

$\mathrm{C}_{\mathrm{t}}$ represents the release LNT concentration at time $\mathrm{t}, \mathrm{V}_{\text {total }}$ represents solution volume, and $\mathrm{Q}_{1}$ represents the total quality of loaded LNT.

Higuchi model: $\mathrm{Q}_{\mathrm{t}}=\mathrm{Kt}^{1 / 2}(4)$

Korsmeyer-Peppas model: $\mathrm{Q}_{\mathrm{t}}=\mathrm{Kt}^{n}(5)$

$\mathrm{Q}_{\mathrm{t}}$ represents the cumulative release rate of LNT until time $\mathrm{t}, \mathrm{K}$ represents the kinetic constant, and $\mathrm{n}$ is an index that reflects the following release mechanisms: Fickian diffusion $(\mathrm{n}<0.43)$, non-Fickian or anomalous diffusion $(0.43<\mathrm{n}<0.85)$, and case II transport $(\mathrm{n}>0.85)$.

\section{Plant treatment with CSL-gel}

50 pieces of CSL-gels or SL-gels were evenly embedded near the root of each plant at approximately four-leaf stage. Each treatment group has twelve repeats. All plants were grown at the same condition with $12 \mathrm{~h}$ of light and $12 \mathrm{~h}$ of darkness at 25 and were given $10 \mathrm{~mL}$ of water every $24 \mathrm{~h}$.

\section{Virus inoculation and visualization of disease symptoms}

All viruses were inoculated 14 days after plants were treated with different hydrogels. The rubbing method was used to inoculate TMV labeled with a green fluorescent protein (GFP) in hydrogel treated plants or CML30 silenced plants. The agroinfiltration was used to inoculate TRV-GFP, TuMV-GFP and PVX in hydrogel treated plants. The infection of virus-GFP in $N$. benthamiana was observed under UV lamp (BlakRay B-100AP, Upland, CA, U.S.A.) at the appropriate time (TMV-GFP: 2 and 7 dpi, TRV-GFP: 5 and 9 dpi, TuMV-GFP: 12 and $16 \mathrm{dpi}$ ). The infection of PVX in $N$. benthamiana was observed under a fluorescent lamp at 18 and $22 \mathrm{dpi}$. At the same time, the inoculated leaves and young leaves of each group were collected in liquid nitrogen and stored at $-80 \operatorname{deg} \mathrm{C}$ for a subsequent experiment.

Quantitative real-time PCR (qPCR) and enzyme-linked immunosorbent assay (ELISA) 
Total RNA was extracted from inoculated leaves and young leaves using RNA extraction kit (Promega Biotechnology Co., Ltd., Shanghai, China). Next, the reverse transcription kit (Shanghai Titan Technology Co., Ltd., Shanghai, China) was used to synthesize cDNA. Quantitative PCR (qPCR) was performed according to the previous method using a CFX Touch Real-time PCR machine (Bio-Rad, USA) and Quantinova SYBR Green PCR Kit (Chongqing Shuguang Biotechnology, China) [66]. Actin was used as an internal reference. Quantification of the relative changes in gene transcript levels was performed using the $2^{-\Delta \Delta^{\prime \prime} \mathrm{T}}$ method. Each sample has three biological replicates. All primers of qPCR were listed in Table $\mathbf{1}$ and S1 . The crude extracts prepared from leaf samples at different inoculation times were used to perform ELISA assay. TMV-GFP antibodies and horseradish substrate coloration were used to detect the protein accumulation of TMV-GFP ${ }^{[29-30]}$.

\section{Construction of $C M L 30$ silencing vector}

$C M L 30 \mathrm{cDNA}$ was amplified from leaf cDNA using primers $C M L-T R V-F$ and $C M L-T R V-\mathrm{R}$ (Table 1 ). The fragment was then inserted into VIGS vector Pash18 at the BamH I and Xhol I site. Finally, the recombinant plasmids were introduced intoAgrobacterium tumefaciens EHA105. The empty vector, TRV-GFP was used for control treatment.

\section{Measurement of plant physiological index after CSL-gel treatment}

A total of 50 pieces of CSL-gel or SL-gel were buried in the soil, and each plant was given $10 \mathrm{~mL}$ of water every day for consecutive 14 days and placed in a constant temperature growth room at $25{ }^{\circ} \mathrm{C}(12 \mathrm{~h}$ of light and $12 \mathrm{~h}$ of darkness). After 14 days, the plant height, plant width, leaf width, fresh weight, and dry weight of $N$. benthamiana were measured. Each treatment group has twelve replicates.

\section{Biosafety evaluation of CSL-gel}

10 crucian carp with a length of 3-4 cm were placed into $1 \mathrm{~L}$ of pure water $(\mathrm{pH}=5.5)$ with different amounts of CSL-gel (the number of CSL-gel pieces per $1 \mathrm{~L}$ of water was 50,100, 200, and 400). Pure tap water was used as a blank control, and each group has three repeats. The life activity and death of crucian carp were observed at $24 \mathrm{~h}$ intervals. Each treatment group has four replicates.

\section{Statistical analysis}

All experimental data presented here involved at least three replicates. The data are presented as means and standard deviations. The statistical analysis was performed with SPSS software (version 17.0) and OriginPro 8.

\section{Results}

\section{Synthesis of CSL-gel and characterization of chitosan core-shell}

To obtain the sustained release of lentinan and calcium ions, we developed a novel and multiple-functional hydrogel by cross-linking the mixed solution of sodium alginate and lentinan with calcium ions to form a hydrogel precursor (SL-gel). A chitosan shell was built on the surface of SL-gel to form a lentinan-loaded hydrogel with a core-shell structure (CSL-gel) (Figure 1a), which is expected to provide the stable and sustained release of lentinan (LNT) and calcium ions (Figure 1b ). To assess the formation of chitosan shell on the surface of CSL-gel, scanning electron microscopy (SEM) and Zeta potential were employed. SEM images of SL-gel and CSL-gel were illustrated inFigure 1c. SEMs showed that the surface of the SL-gel with large pores was loose, but a dense chitosan shell covering the SL-gel was observed on the surface of CSL-gel (Figure 1c ). Moreover, the elemental analysis showed that elemental nitrogen was detected on the surface of the CSL-hydrogel (Table 2 ), indicating that chitosan was added on the surface of CSL-gel. To avoid the influence of LNT on the FTIR and zeta analysis, we performed the Fourier transform infrared spectrometry (FTIR) and zeta analysis using the hydrogel without LNT to characterize the chitosan shell. Figure 1dshowed a shared peak located at approximately $3340 \mathrm{~cm}^{-1}$ was observed in the FTIR spectrum of the S-gel and CS-gel. However, the CS-gel features a fully developed peak on $3340 \mathrm{~cm}^{-1}$, which is attributed to the overlap associated with the peaks of the - $\mathrm{OH}$ stretching vibration of $\mathrm{SA}$ and the $-\mathrm{NH}$ stretching vibration 
of the chitosan in comparison to the S-hydrogel. Besides, the CS-gel exhibited a peak at approximately 2927 $\mathrm{cm}^{-1}$, which was formed by the C-H stretching vibration of chitosan, suggesting that the reaction of adding chitosan to the S-gel was successful. Sodium alginate is composed of carbon, hydrogen, and oxygen, and the surface is negatively charged. The zeta potential analysis showed that the surface of CS-gel has a positive charge, but the S-gel with a negative charge (Figure 1e ). Taken together, our findings strongly indicate that a chitosan shell is added to the SL-gel, which has the potential to stabilize the structure of CSL-gel and provide the sustained release of LNT and calcium ions.

\section{Sustainable release of LNT and calcium ion of CSL-gel}

To assess the release property of loaded drugs in CSL-gel, we measured the release rate of LNT at the corresponding time according to the regression equation of LNT $\left(\mathrm{y}=0.0028 \mathrm{x}+0.0266, \mathrm{R}^{2}=0.9998\right.$, Figure S1 ). As shown in Figure 2a and2b, CSL-gel maintained a stable and cumulative release of LNT from the first day to the 14 days after soaking. However, a substantial amount of LNT was released from SL-gel on the first day and the LNT release slightly increased with the extension of soaking time and reached the maximum amount on the eighth day after soaking. At the ninth day, a limited amount of LNT was released following the extension of the soaking. Furthermore, we used Korsmeyer-Peppas and Higuchi models to determine the release of LNT. Figure 2c showed that a clear linear correlation $\left(\mathrm{R}^{2}=0.989\right)$ between the cumulative release rate and the logarithm of time from CSL-gel in the Korsmeyer-Peppas model fitting curve was observed. Similarly, a high linear correlation between the cumulative release rate and the square root of time during the release of LNT from the CSL-gel $\left(\mathrm{R}^{2}=0.9823\right)$ was also observed in the Higuchi model fitting curve (Figure 2d ). However, no linear correlation between the cumulative release rate and the square root of time $\left(R^{2}=0.8683\right)$ and between the cumulative release rate and the logarithm of time $\left(\mathrm{R}^{2}=0.933\right)$ of SL-gel was observed. The corresponding dispersion coefficient (n) of SL-hydrogel was 0.3089 ( $\mathrm{n}<0.43$ ), indicating that the release of LNT from SL-gel under these conditions followed Fickian diffusion (Table 3 ). In contrast, the dispersion coefficient (n) of CSL-gel was $0.6305(0.43<\mathrm{n}<0.85)$, which indicates the CSL-gel followed non-Fick diffusion mechanism (Table 3 ). These findings demonstrated that the chitosan shell controls the release of LNT from the CSL-gel, resulting in the cumulative and sustainable release of LNT.

Soil is a complex system in which the properties, such as $\mathrm{pH}$, temperature, and ion concentration, are dynamic due to the impact of external factors such as fertilization, irrigation, and rainfall ${ }^{[31]}$. The drug release from hydrogel will be influenced by different soil environments. To assess the release rate of LNT at different soil properties, we measured the release of LNT from hydrogel at different $\mathrm{pH}$, temperatures, and ion concentrations. As shown in Figure 2e , the cumulative LNT release rate of CSL-gel highly increased following the increased temperature, which is caused by the high swelling of the hydrogel at a high temperature. Similarly, the corresponding release rate of LNT from CSL-gel largely increased from $7.54 \%$ to $41.35 \%$ when the $\mathrm{pH}$ increased from 3 to 9 (Figure 2f ). This observation follows the fact that at low $\mathrm{pH}$ most of the carboxyl groups on alginate are deionized and form strong hydrogen bonds with hydroxyl groups, resulting in a tight shrinkage of the calcium alginate pellet that prevents the LNT release from the CSL-gel. Interestingly, we found that ion concentration present positively regulated the release LNT from CSL-gel. As shown in Figure 2e , the cumulative release rate of LNT highly increased from $33.7 \%$ to $99.5 \%$ following the increased $\mathrm{Na}^{+}$concentration from 0 to $0.8 \mathrm{M}$. The ion exchange between $\mathrm{Na}^{+}$and $\mathrm{Ca}^{2+}$ strengthens the electrostatic repulsion between - $\mathrm{COO}^{-}$, which ultimately increases the swelling of the hydrogel to stimulate the release of LNT ${ }^{[32]}$.

\section{CSL-gel promotes plant growth and enhances the resistance against plant virus}

The chitosan present on the surface of CSL-gel can form a robust electrostatic force with sodium alginate, resulting in the accelerated release of calcium ions from the gel. To test our hypothesis, we soaked the SL-gel or CSL-gel in water and measured the concentration of $\mathrm{Ca}^{2+}$ in the water solution at 2-day intervals. As shown in Figure 3a, the amount of $\mathrm{Ca}^{2+}$ released from the CSL-gel was significantly higher than that of SL-gel. To assess whether the released $\mathrm{Ca}^{2+}$ can promote plant growth and development, we measured the plant height, leaf width, dry weight, and fresh weight of $N$. benthamiana at 14 days after SL-gel or 
CSL-gel treatment. Figure 3b-g showed CSL-gel-treated plants displayed a significant increase in plant growth, including height, plant width, leaf width, dry weight compared to that of the water-treated plant. In addition, the growth indexes of plants treated with CSL-gel were higher than those of the S-gel-treated and control plants (Figure 3b-g ). Moreover, After 30 days of treatment, the growth trend of CSL-gel-treated plants was significantly higher than that of the water-treated and SL-gel-treated group (Figure S2 ). These results demonstrated that CSL-gel promotes plant development.

To examine the anti-virus activity of the CSL-gel with the sustained release of LNT and calcium ion, we applied the CSL-gel into the soil surrounding the plant root. SL-gel serves as a control. We inoculated the TMV-GFP by rubbing on the leaves of the plant at 7 day after CSL-gel application. As shown in Figure $3 \mathbf{h}$, at 2 dpi the number of green fluorescent spots in CSL-gel-treated $N$. benthamiana was the same as that on the SL-gel-treated plants. At $7 \mathrm{dpi}$, the green fluorescent signals present in the young leaves of the CSL-gel-treated plant were significantly weaker than that of the water and SL-gel treatment groups (Figure 3h ). qPCR analysis showed that the number of TMV-CP transcripts between the two treatment groups was no significant differences, but at 7 dpi the expression level of TMV- $C P$ in the CSL-gel-treated plant was significantly lower than that in the SL-gel-treated and control plants (Figure 3i-j ). Taken together, we can conclude that the CSL-gel with the sustainable release of LNT and calcium ion significantly enhances the tobacco resistance against TMV as well as promoting the plant development.

\section{CSL-gel induces CML30 expression and silencing ofCML30 enhances the susceptibility to TMV}

The increased $\mathrm{Ca}^{2+}$ concentration in plant cells promotes the expression of $C M L$ that plays a role in plant development and resistance in response to biotic stresses ${ }^{[33]}$. To examine the impact of CSL-gel on the expression of $C M L$ genes, the relative expression of CML30in plants treated with CSL-gel was quantified by qPCR. SL-gel treated plant severs as a positive control. Figure $4 \mathbf{a}$ and 4 bshowed that the CML30 expression in the CSL-gel treated plants was significantly higher than that in the SL-gel treated plants at 2 and 7 days after treatment.

To further determine whether upregulated $C M L 30$ expression by CSL-gel contributes to the TMV resistance, we generated the CML30RNAi silencing construct to transiently knockdown the expression of $C M L 30$ in tobacco by agroinfiltration. As shown in Figure 4c, PCR analysis showed that the expected silencing fragment was present in the tested 5 Agrobacterium strains. qPCR analysis showed that $C M L 30$ expression level in the silenced plant was $80 \%$ reduced compared with the wild-type plant at 8 days after agroinfiltration (Figure 4e ), indicating that the silencing construct is effective in reducing CML30 expression. Eight days after infiltration, all infiltrated leaves were inoculated with TMV-GFP.Figure $\mathbf{4 d}$ showed that at $3 \mathrm{dpi}$, the GFP signals increased compared to that at 2 dpi, but the level of signals present in silenced plants and WT plants were similar. At 4 dpi, strong GFP signals were observed in the inoculated leaves of silenced plants compared with that in the WT plants and in the young leaves of the silenced plant increased GFP signals were visualized, while in WT plant limited GFP signals were observed in the young leaves (Figure 4d ). At $5 \mathrm{dpi}$, pronounced green fluorescence signals were visualized in the young leaves of the silenced plant, but slightly expanded GFP signals were observed in the young leave of WT plant (Figure 4d ). In addition, the number of TMV- $C P$ transcripts was quantified by qPCR. Figure $\mathbf{4 f}$ showed that the expression of TMV- $C P$ in the silenced plant was significantly higher than that in the WT plant at 3,4 and 5 dpi. Similarly, ELISA analysis showed that a significant amount of TMV was accumulated in the silenced plant compared to that in the WT plant (Figure 4g ). Based on our findings, we can conclude that silencing CML30 promotes the TMV infection on N. Benthamian, indicating that CSL-gel induces CML30 expression that contributes to the TMV resistance.

\section{Anti-TMV activity of CSL-gel mainly depends on the chitosan shell and calcium ion}

CSL-gel promotes the expression of $C M L 30$ and the silencing of CML30 enhances TMV infection. To further determine that CML30 induced by calcium ion released from the CSL-gel contributes to the resistance against TMV infection, we generated the CS-gel and S-gel without LNT to eliminate the role of LNT on the anti- 
TMV activity. As shown in Figure 5a, at 2 dpi, the number of green fluorescent spots in the inoculated leaves of $N$. benthamiana treated with CS-gel was lower than that of the S-gel and water-treated plants. qPCR analysis showed that the expression levels of TMV-CP were significantly lower in the inoculated leaves of $N$. benthamiana treated with CS-gel than that in the water and S-gel-treated plants (Figure 5b and 5c ). At $7 \mathrm{dpi}$, the green fluorescence signals and the number of TMV-CP transcripts of the water control group and S-gel treatment group were significantly higher than that of CS-gel control. However, there was no significant difference between the water-treated and the S-gel-treated plans on the TMV infection at 2 and $7 \mathrm{dpi}$. These findings indicated that CML30induced by CSL-gel also plays an important role in anti-TMV.

\section{CSL-gel induces broad-spectrum resistance against different plant virus}

To assess whether CSL-gel induces broad-spectrum resistance against different virus, $N$. benthamiana plants treated with CSL-gel were separately inoculated with the tobacco rattle virus (TRV), potato virus X (PVX) and turnip mosaic virus (TuMV) by rubbing. SL-gel and water treated plants were included as controls. At the early stage of infection (TRV: $5 \mathrm{dpi}$, TuMV: 12dpi), the number of green fluorescent spots in CSL-geltreated $N$. benthamiana was similar to SL-gel-treated plants. However, in the late stage of infection (TRV: $9 \mathrm{dpi}$, TuMV: $16 \mathrm{dpi}$ ), the green fluorescent signals present in the young leaves of the CSL-gel-treated plant were significantly lower than that of the water and SL-gel treatment groups (Figure 6a-b ). Figure 6c showed that 18 days after inoculation, symptoms caused by PVX were relatively mild in the young leaves of and CSL-gel-treated plants, while PVX caused severe symptoms in the young leaves of water-treated plants. At 22 dpi, PVX infection developed severely curly young leaves in the water-treated plants, and plants treated with SL-gel showed mild symptoms. However, no symptoms caused by PVX infection were observed in the CSL-gel-treated plants (Figure 6c ). Furthermore, qPCR analysis showed that the number of virus-CP transcripts in the CSL-gel-treated plant was significantly lower than that in the SL-gel and water-treated plants (Figure 6d-i ). Therefore, we can conclude that CSL-gel inhibits the infection caused by the majority of viruses in $N$. benthamianaplants.

\section{Safety test of CSL-gel}

The toxicity test of pesticides on aquatic organisms is an important measure strategy for the safety evaluation of pesticides ${ }^{[34]}$. Our previous studies showed that the $\mathrm{LC}_{50}$ of LNT to crucian carp at $24,48,72$, and 96 $\mathrm{h}$ was about $1.6,0.8,0.4$, and $0.4 \mathrm{mg} / \mathrm{mL}$, respectively ${ }^{37}$. To evaluate the CSL-gel safety, the number of deaths of crucian carp was examined in the water immersed with series of numbers of CSL-gel at $24 \mathrm{~h}, 48 \mathrm{~h}$, $72 \mathrm{~h}$ and $96 \mathrm{~h}$. The pure tap water was used as a negative control. As shown inTable 4 , no dead crucian carp was observed in the CSL-treated water with LNT concentration lower than $336 \mathrm{mg}$ as well as in the water control. However, dead crucian carp was observed at $48 \mathrm{~h}$ in the water treated with 400 pieces of CSL-gel with an LNT concentration at $336 \mathrm{mg}$, indicating that CSL-gel maintained a relatively high safety to environmental organisms.

\section{Discussion}

Plant viruses cause a common systemic disease named plant cancers that differ from other plant diseases [1-2]. They can entirely rely on the host plant to acquire nutrients for replication, movement, and other life activities, resulting in damage to plant chloroplasts and photosynthesis, reduced accumulation of plant carbohydrates, and inhibiting plant growth ${ }^{[1-3]}$. Traditional methods depend on virus deactivators and plant immunity inducers to minimize the infection ${ }^{[35]}$. However, most virus deactivators and plant immunity inducers are not able to completely inhibit the proliferation and movement of the virus in the plant after the virus infection ${ }^{[3]}$. In this study, a lentinan-loaded hydrogel with the core-shell structure was developed, which features the stable and sustainable release of lentinan and calcium ions. The prolonged-release of LNT and calcium ions significantly promotes plant growth and development and provides broad-spectrum resistance against TMV, TuMV, PVX and TRV. In addition, we found that the sustained release of calcium ions from the CSL-gel activates the expression of calmodulin-like protein 30 (CML30), and the silencing of CML30 enhances the susceptibility of tobacco to TMV. We summarize the action of CSL-gel in Figure 
7. Therefore, the low-cost and easily synthesized CSL-gel with a novel mode of action triggering CML30 expression has the potential to be utilized in the field against severe virus disease and increase the yield of crop plants.

Our results showed that the chitosan shell prevents the rapid bursting of the hydrogel, resulting in the controlled and stable release of LNT and calcium ions and the extended-release time (Figures $\mathbf{1}$ and $\mathbf{2}$ ). Polysaccharide immunity inducer, such as amino oligosaccharides, chitosan, chitin, and lentinan, has been widely used in anti-plant virus diseases ${ }^{[36]}$. Lentinan, as a biological polysaccharide, has multiple disease-resistant functions, such as closing plant stomata, inducing the expression of plant resistance genes and improving plant disease-related enzyme activity ${ }^{[25]}$. However, previously generated hydrogel loaded with LNT is confined in field application due to the limited induction time of lentinan and its instability in the complex field environment ${ }^{[25]}$. The newly synthesized hydrogel with a core-shell structure that functions as a slow-release carrier prolongs the action time of lentinan on plants, promotes the plant growth and induces the resistance of $N$. benthamiana to TMV (Figure 3 ). At present, plant immunity inducers as an alternative agent are more effective in controlling plant virus disease ${ }^{[35]}$. Many studies reported that plant immunity inducers can induce the expression of plant disease-related genes, and promote the activities of stress-related enzymes to comprehensively improve the resistance of plants to pathogens ${ }^{[35]}$. For example, Lentinan has been used as a common inducer in the prevention and treatment of plant virus diseases in the field. It can significantly promote the activity of SOD, POD and CAT and stimulate the expression of $P R 1$ and $P R 3$ to improve plant resistance ${ }^{[36-37]}$. Previous studies showed that chitosan was able to increase the activity of plant defense-related enzymes (PAL, PPO, POD, CAT, SOD), and induce the production of secondary metabolites associated with disease resistance and induce phenolic metabolic pathways to improve the antagonism of plants to fungi, bacteria and viruses ${ }^{[38-39]}$.

Calcium ion $\left(\mathrm{Ca}^{2+}\right)$, as one of the important nutrient elements, can increase the germination rate of plant seeds, promote the development of plant roots and leaves, and increase the absorption of nutrients to enhance plant growth ${ }^{[7,40]}$. Previous studies showed that the application of calcium ion at a concentration in the range of $0-14 \mathrm{mmol} / \mathrm{L}$ increases plant growth, root growth, and dry matter accumulation ${ }^{[40]} \cdot \mathrm{Ca}^{2+}$, as a universal second messenger, can regulate the plant response to biotic and abiotic stresses. For example, it can regulate plant cell membrane protective enzyme systems to alleviate the effects of drought, salt stress, and water stress on growth ${ }^{[41-42]}$. The activities of antioxidant enzymes, such as SOD, POD, and CAT, in soybeans and apples treated with calcium ions were significantly improved ${ }^{[43]}$. Furthermore, the application of calcium fertilizer effectively prevents the occurrence of brown spot disease ${ }^{[44]}$. Our results showed that the chitosan shell promotes the CSL-gel to stably and sustainably release $\mathrm{Ca}^{2+}$ owing to the strong electrostatic interaction between SA and chitosan (Figure 2 ). The plants treated with CS-gel exhibit significantly enhanced growth than those treated with the SL-gel (Figures 3 and $\mathbf{5}$ ). This evidence is consistent with previous reports, indicating that CSL-gel with the stable and sustainable release of calcium ions promotes plant growth and improves plant resistance against viruses.

Previous studies showed that $\mathrm{Ca}^{2+}$ is perceived by calmodulin $(\mathrm{CaM})$ and calmodulin-like $(C M L)$ proteins to participate in physiological and biochemical reactions in plants ${ }^{[8-9]}$. As a type of plant-specific $\mathrm{Ca}^{2+}$ receptor, $C M L$ involves various physiological activities in the process of plant growth and development, such as regulating plant defense responses, enhancing plant anti-stress responses, and controlling plant hormone levels ${ }^{[22,45-46]}$. For example, overexpression of Arabidopsis CML8confers enhanced resistance to Pseudomonas syringae in an SA-dependent process ${ }^{[47]}$. ArabidopsisCML9 is rapidly and strongly induced by Pseudomonas syringae and abiotic stress and abscisic acid (ABA), which acts as a positive regulator in response to Pseudomonas syringae and a negative regulator to salt stress ${ }^{[4-49]}$. Tomato plants overexpressing $C M L 44$ exhibit higher antioxidant enzyme activity and greater tolerance to abiotic stresses ${ }^{[2]}$. Our results showed that CSL-gel strongly induces the expression of CML30 and the silencing of CML30 enhances the infection of TMV (Figure 4 ), suggesting that CML30 plays a positive role in the resistance to TMV. The CSL-gel maintains a stable and cumulative release of calcium ions into the soil, which activates plant CML30 to adapt to the increased calcium ions in the environment. In turn, the accelerated expression of CML30 enhances the resistance against TMV. However, the mechanisms underlying this observation remain 
unknown. Because $C M L$ as a sensor of $\mathrm{Ca}^{2+}$ regulates diverse plant processes, the identification of $C M L 30$ interactors will aid us in understanding the anti-virus activity of $C M L 30$ in the subsequent studies.

\section{Conclusions}

A hydrogel with a core-shell structure and sustainable release of lentinan and calcium ions based on the polycationic properties of chitosan was developed in this study. This hydrogel has multiple functions including the promotion of plant growth, continuous induction of plant resistance against the different virus, and enhancement of CML30 expression to improve TMV resistance. These findings presented here form a solid basis for us to understand the resistance against TMV, highlight the importance of $C M L$ in plants against TMV infection and expand our tools to control plant virus diseases as well in the future.

\section{Author contributions}

S. X., X. C., J .W., L. M. and X. S. conceived and designed the experiments. S.X., X. C., J. W., H. P., X. L., L. J., Z. C., J. H. performed the experiments, S. X., X.C., J. W., D. W., L.M., and X.S. analyzed the data. S.X., L.M. and X.S.drafted the manuscript.

\section{Funding}

This study was partly supported by the National Natural Science Foundation of China (31670148 and 31870147), the Fundamental Research Funds for the Central Universities (XDJK2020B064 and XDJK2017C015), the Science and Technology Projects of Chongqing Company of China Tobacco Corporation (NY20180401070001, A20201NY02-1306 and NY20180401070010).

\section{Conflict of interest statement}

The authors declare no competing financial interest.

\section{References}

1. Hammerschmidt R., J P Metraux, Loon L C V.(2001). . Inducing Resistance: A Summary of Papers Presented at the First International Symposium on Induced Resistance to Plant Diseases, Corfu, May 2000[J]. European Journal of Plant Pathology, 107(1).

2. Buzayan J M., Gerlach W L , Bruening G. (1986). . Non-enzymatic cleavage and ligation of RNAs complementary to a plant virus satellite RNA[J]. Nature, 323(6086):349-353.

3. Rani M., Sharma S, Chauhan R. (2017). Synthesis, Characterization and Antibacterial Evaluation of Some Azole Derivatives. Indian Journal of Pharmaceutical Education and Research 51: 650-655.

4. Yamakawa., H. (1998). Spermine Is a Salicylate-Independent Endogenous Inducer for Both Tobacco Acidic Pathogenesis-Related Proteins and Resistance against Tobacco Mosaic Virus Infection[J]. Plant Physiology. 118(4):1213-1222.

5. Xiang, Y., Wang, N. Song, J.( 2013). Micro-nanopores fabricated by high-energy electron beam irradiation: Suitable structure for controlling pesticide loss. J. Agric. Food Chem, 61 (22), 5215-5219.

6. Lin K I., Kao Y Y. Kuo H K, et al.(2006). Reishi Polysaccharides Induce Immunoglobulin Production through the TLR4/TLR2-mediated Induction of Transcription Factor Blimp-1[J]. Journal of Biological Chemistry, 281(34):24111-24123.

7. Tang R J., Liu H, Yang Y, et al. (2012). Tonoplast calcium sensors CBL2 and CBL3 control plant growth and ion homeostasis through regulating V-ATPase activity in Arabidopsis[J]. Cell Research, 22(12):16501665 .

8. Batisti O, Kudla J. (2012). Analysis of calcium signaling pathways in plants[J]. Biochimica Et Biophysica Acta General Subjects, 2012, 1820(8): 1283-1293. 
9. DeFalco T A, Bender K W, Snedden W A. (2010). Breaking the code: Ca^s sensors in plant signaling $[\mathrm{J}]$. biochemical journal, 425(1):27-40.

10. Braam J., Davis RW. (1990). Rain-, wind-, and touch-induced expression of calmodulin and calmodulinrelated genes in Arabidopsis. Cell, 60 (3): 357-364.

11. Bongkoj., Boonburapong T, Buaboocha. (2007). Genome-wide identification and analyses of the rice calmodulin and related potential calcium sensor proteins[J]. Bmc Plant Biology, 7: 4.

12. Bender K W., Snedden W A. (2013). Calmodulin-Related Proteins Step Out from the Shadow of Their Namesake[J]. Plant Physiology, 163(2):486-495.

13. Nakaham K SM., asuta C, Yamada S, et a1. (2012). Tobacco calmodulin like protein provides secondary defse by binding to and directing degradation of vims RNA silencing suppressors[J].Pmc Nad Acad Sci USA, 109: $10113-10118$.

14. Roberts D M., Harmon A C. (1992). Calcium-Modulated Proteins: Targets of Intracellular Calcium Signals in Higher Plants[J]. Annual Review of Plant Physiology and Plant Molecular Biology, 43(1):375-414.

15. Mcainsh M R., Pittman J K. (2019). Shaping the calcium signature[J]. New Phytologist, 181(2):275-294.

16. Ma W., Smigel A, Tsai Y C, et a1. (2008). Innate immunity signaling:cytosolic $\mathrm{Ca}^{2+}$ elevation is 1inked to downstream nitric oxide generation through the action of calmodulin or a calmodulin-lik8 protein [J]. Plant Physiol, 148: 818-828.

17. Xu B., Cheval C, Laohavisit A, et al. (2017). A calmodulin-like protein regulates plasmodesmal closure during bacterial immune responses. New Phytol, 215 (1): 77.

18. Leba L J, Cheval C, Inmaculada O M, et al. (2012). CML9, an Arabidopsis calmodulin-like protein, contributes to plant innate immunity through a flagellin-dependent signalling pathway[J]. The Plant Journal, 71(6):976-989.

19. Nakahara K S., Masuta C, Yamada S, et al. (2012). Tobacco calmodulin-like protein provides secondary defense by binding to and directing degradation of virus RNA silencing suppressors. Proc Natl Acad Sci USA, 109 (25): 10113-10118.

20. Zhang, Q Y., Liu, X J, Liu X, et al. (2017). Genome-Wide Identification, Characterization, and Expression Analysis of Calmodulin-Like Proteins (CMLs) in Apple[J]. Horticultural Plant Journal, 3( 6):219231.

21. Asamura S., Mochizuki Y, Yamamoto M, et al. (2010). Bone Regeneration Using a Bone Morphogenetic Protein-2 Saturated Slow-Release Gelatin Hydrogel Sheet[J]. Annals of Plastic Surgery, 64(4):496-502.

22. Builders P F., Kunle O O, Okpaku L C, et al. (2008). Preparation and evaluation of mucinated sodium alginate microparticles for oral delivery of insulin[J]. European Journal of Pharmaceutics and Biopharmaceutics, 70(3):0-783.

23. Nokhodchi A., Tailor A. In situ cross-linking of sodium alginate with calcium and aluminum ions to sustain the release of theophylline from polymeric matrices[J]. Farmaco, 2004, 59(12):999-1004.

24. Mai B J., Jia M Q, Liu S P, et al. (2020). Smart Hydrogel-Based DVDMS/bFGF Nanohybrids for Antibacterial Phototherapy with Multiple Damaging Sites and Accelerated Wound Healing, ACS Applied Materials \& Interfaces, 10156-10169.

25. Xiang S Y., Lv X, He L H, et al. (2019). Dual-Action Pesticide Carrier That Continuously Induces Plant Resistance, Enhances Plant Anti-Tobacco Mosaic Virus Activity and Promotes Plant Growth. Journal of Agricultural and Food Chemistry. 67, 10000-10009.

26. Gao C., Liu M, Chen J, et al. (2009). Preparation and controlled degradation of oxidized sodium alginate hydrogel[J]. Polymer Degradation and Stability, 94(9):1405-1410. 
27. Lin Y H., Sonaje K, Lin K, et al. (2008). Multi-ion-crosslinked nanoparticles with pH responsive characteristics for oral delivery of protein drugs. J. Controlled Release 132, 141-149.

28. Korsmeyer R W., Gurny R, Doelker E, et al. (1983). Mechanisms of solute release from porous hydrophilic polymers. Int. J. Pharm. 15 (1), 25-35.

29. Ma L S., Djavaheri M, Wang HY, et al., (2018). Leptosphaeria maculans effector protein AvrLm1 modulates plant immunity by enhancing MA P kinase 9 phosphorylation. iScience, 3:177-191

30. Peng H R, Pu Y D, Yang X, et al. (2019). Overexpression of a pathogenesis-related gene NbHIN1 confers resistance to Tobacco Mosaic Virus in Nicotiana benthamiana by potentially activating the jasmonic acid signaling pathway. Plant Science, 283 : 147-156.

31. Reth S., Reichstein M, Falge E. (2005). The effect of soil water content, soil temperature, soil pH-value and the root mass on soil $\mathrm{CO}^{2}$ efflux-A modified model. Plant Soil, 268 (1), 21-33.

32. Xiang Y, Zhang G C, Liu B, et al. (2018). Fabrication a pH-responsively controlled-release pesticide using an attapulgite-based hydrogel. ACS Sustainable Chem. Eng. 6 (1), 1192-1201.

33. Kwang-Moon Cho, Nguyen H T K, Kim S Y, et al. (2016). CML10, a variant of calmodulin, modulates ascorbic acid synthesis[J]. New Phytologist, 209: 664-678.

34. Tian W J, Zhuang T Y, Cheng S L, et al. (2005). The acute toxicity of pyragne, an insecticide for control of Solenopsis invicta, to sword fish (Xiphophorus helleri). Entomol. Knowl. 42 (6), 650-653.

35. Ryu S M, Lee H M, Song E G, et al. (2017). Antiviral Activities of Trichothecenes Isolated from Trichoderma albolutescens against Pepper Mottle Virus[J]. Journal of Agricultural \& Food Chemistry, 65(21):42734279 .

36. Zhao, X., She, X. Du, Y. et al, (2007). Induction of antiviral resistance and stimulary effect by oligochitosan in tobacco. Pestic.Biochem. Physiol. 87 (1), 78-84.

37. Yin, H., Zhao, X. Du, Y. (2010). Oligochitosan: A plant diseases vaccine-A review. Carbohydr. Polym. $82(1), 1-8$.

38. Orzali L , Forni C , Riccioni L. (2010). An overview of the plant response to pathogen attack: chitosan as a general elicitor of induced resistance in plants[J]. Minerva Biotecnologica, 22(1/2):55-56.

39. Benhamou N., Georges T. (1992). Treatment with chitosan enhances resistance of tomato plants to the crown and root rot pathogen Fusarium oxysporum f. sp. radicis-lycopersici[J]. Physiological \& Molecular Plant Pathology, 41(1):33-52.

40. Chowdhury S R., Choudhuri M A. (1967). Effects of calcium ions on responses of two jute species under water-deficit stress[J]. Physiologia Plantarum, 1986, 68(1):86-92.

41. Jones R G W., Lunt O R. (1998). The function of calcium in plants[J]. 33(4):407-426.

42. Xu H. (1998). Role of Calcium in Signal Transduction during the Hypersensitive Response Caused by Basidiospore-Derived Infection of the Cowpea Rust Fungus[J]. The plant cell online, 10(4):585-598.

43. Munir S., Liu H, Xing Y, et al. (2016). Overexpression of calmodulinlike (ShCML44) stress-responsive gene from Solanum habrochaites enhances tolerance to multiple abiotic stresses. Science Report, 6: 31772.

44. Wu XM., Qiao Z, Liu HP, et al. (2017). CML20, an Arabidop-sis calmodulin-like protein, negatively regulates guard cell ABA signaling and drought stress tolerance. Front Plant Science, 8: 824.

45. Min C K, Chuang W S, Yun D J, et al. (2009). Calcium and Calmodulin-Mediated Regulation of Gene Expression in Plants[J]. Molecular Plant, 2: 13-21.

46. Zhu X Y, Robe E, Lucile J, et al. (2017). CML8, an Arabidopsis Calmodulin-Like Protein, Plays a Role in Pseudomonas syringae Plant Immunity[J]. Plant \& Cell Physiology, 58(2): 307-319. 
47. Xiaoyang Z , Robe Eugenie, Lucile J , et al. (2016). CML8, an Arabidopsis calmodulin-like protein plays a role in Pseudomonas syringae plant immunity[J]. Plant and Cell Physiology, (2):2.

48. Louis-Jerme L, Cecilia C, Inmaculada O M, et al. (2012). CML9, an Arabidopsis calmodulin-like protein, contributes to plant innate immunity through a flagellin-dependent signalling pathway.[J]. Plant Journal, 71(6):976-989.

49. Magnan F, Ranty B , Charpenteau M, et al. (2008). Mutations in AtCML9, a calmodulin-like protein from Arabidopsis thaliana, alter plant responses to abiotic stress and abscisic acid[J]. The Plant Journal, 56(4):575-589.

\section{Legends:}

Figure 1. Synthesis of CSL-gel and characterization of chitosan shell . (a). schematic representation of the synthesis of CSL-gel. (b). Diagram of CSL-gel and SL-gel drug release simulation. (c). SEM images of surface composition of SL-gel and CSL-gel. (d). Zeta potential of the S-gel without LNT and chitosan shell and CS-gel without LNT. (e). FTIR spectra of the S-gel and CS-gel.

Figure 2. CSL-gel exhibits sustainable and cumulative release of LNT . (a). Comparison of the cumulative release of LNT from the SL-gel and CSL-gel. (b). The cumulative release rate of LNT from CSL-gel and SL-gel. (c). The fitting curves for the Korsmeyer-Peppas model. (d). The fitting curves for the Higuchi model. (e). The cumulative release rate of LNT from CSL-gel at different temperatures. (f), The cumulative release rate of LNT from CSL-gel at different $\mathrm{pH}$. (g). The cumulative release rate of LNT from CSL-gel at different $\mathrm{Na}^{+}$concentration. Double asterisks indicate separation among CSL-gel and SLgel at the same amount by Duncan's multiple comparisons $(* *, p<0.01)$. Vertical bars indicate standard deviations $(\mathrm{n}=3)$.

Figure 3. CSL-gel with the sustainable release of calcium ions promotes plant growth and significantly enhances the resistance of $N$. benthamiana against TMV . (a). Comparison of calcium ions cumulative release between SL-gel and CSL-gel. (b-f).Comparison of plant height, plant width, leaf width, dry weight, and fresh weight between CSL-gel and SL-treated plants. (g). Representative pictures showing the plant growth between different treatments. (h). CSL-gel treated $N$. benthamiana plants display enhanced resistance against TMV. $N$. benthamiana plants were inoculated with TMV-GFP constructs by rubbing and representative pictures were photographed at 2 and 7 dpi. (i) qPCR analysis showing the expression level of $T M V-C P$ in the inoculated leaves of $N$. benthamiana at 2 dpi. (j). qPCR analysis showing the expression level of TMV-CP in the inoculated leaves of $N$. benthamiana at 7 dpi. The expression level was normalized to Actin. Mean values displayed in each bar followed by different letters, are significantly different according to Duncan's multiple range test $(\mathrm{p}<0.05)$.

Figure 4. CSL-gel triggers CML30 expression and silencing CML30 increases TMV infection in N. benthamiana .(a-b). CSL-gel treatment triggers the expression of CML30 at 2 days and 7 days. (c). The PCR results showing the presence of recombinant plasmids in Agrobacterium. (d). Silencing CML30increases TMV infection. The leaves of $N$. benthamiana were inoculated with TMV-GFP by rubbing. The green GFP fluorescence signals were visualized at 2, 3, 4 and 5 dpi under UV light. Representative pictures are showed. (e). qPCR analysis showing the relative expression of CML30 in the silenced $N$. benthamiana. (f). qPCR analysis showed the expression level of TMV-CP in the inoculated leaves of $C M L 30$ silenced $N$. benthamiana at 3, 4 and 5 dpi. (g). The accumulation of TMV-CP protein was detected by ELISA.

Figure 5. Comparison of the anti-TMV activity of S-gel and CS-gel. (a). N. benthamiana plants treated with CS-gel display increased resistance against TMV. The leaves of $N$. benthamianawere inoculated with TMV-GFP by rubbing. The green GFP fluorescence signals were visualized at 2 and 7 dpi under UV light. Representative pictures are showed. (b). qPCR analysis showing the relative expression of $T M V$ - $C P$ in the inoculated leaves of $N$. benthamiana at 2 dpi. (c). qPCR analysis showed the expression level of $T M V-C P$ in the young leaves of $N$. benthamiana at $7 \mathrm{dpi}$.

Figure 6. CSL-gel treated $N$. benthamiana plants exhibit significantly increased resistance 
against TRV, TuMV and PVX. The leaves of $N$. benthamiana were inoculated with TRV, TuMV and PVX by rubbing or agroinfiltration 14 days after CSL-gel treatment. (a). The green GFP fluorescence signals were visualized at 5 and 9 days after TRV inoculation under UV light. Representative pictures are showed. (b). The green GFP fluorescence signals were visualized at 12 and 16 days after TuMV inoculation under UV light. Representative pictures are showed. (c). Disease symptoms caused by PVX were observed at 18 and 22 days after inoculation and representative pictures are showed. (d-e). qPCR analysis showing the relative expression of $T R V-C P$ in the inoculated leaves of $N$. benthamiana at 5 dpi and in the young leaves at 9 dpi. (f-g). qPCR analysis showing the relative expression of $\mathrm{T} u M V V$ - $C P$ in the inoculated leaves of $N$. benthamiana at $12 \mathrm{dpi}$ and in the young leaves at $16 \mathrm{dpi}$. (h-i). qPCR analysis showing the relative expression of $P V X-C P$ in the inoculated leaves of $N$. benthamiana at $18 \mathrm{dpi}$ and in the young leaves at 22 dpi.

Figure 7. Schematic representation of a proposed action model of CSL-gel on plants to improve broadspectrum resistance to different viruses.

Table 1.Primers used in this study.

Table 2. Elemental analysis of the SL-gel and CSL-gel.

Table 3. Kinetic parameters associated with LNT released from the SL-gel and CSL-gel.

Table 4. Toxicity of CSL-gel to Crucian Carp.

\section{Hosted file}

Table and Figure.pdf available at https://authorea.com/users/374560/articles/492070-alentinan-loaded-hydrogel-with-core-shell-structure-induces-broad-spectrum-resistanceagainst-plant-virus-by-activating-the-expression-of-cml30 